\title{
PRODUCING MAGNETAR MAGNETIC FIELDS IN THE MERGER OF BINARY NEUTRON STARS
}

\author{
Bruno Giacomazzo $^{1,2}$, Jonathan Zrake ${ }^{3}$, Paul C. Duffell ${ }^{4}$, Andrew I. MacFadyen ${ }^{4}$, and Rosalba Perna ${ }^{5}$ \\ 2 Physics Department, University of Trento, via Sommarive 14, I-38123 Trento, Italy \\ 2 INFN-TIFPA, Trento Institute for Fundamental Physics and Applications, via Sommarive 14, I-38123 Trento, Italy \\ ${ }^{3}$ Kavli Institute for Particle Astrophysics and Cosmology, Stanford University, Menlo Park, CA 94025, USA \\ ${ }^{4}$ Center for Cosmology and Particle Physics, Physics Department, New York University, New York, NY 10003, USA \\ ${ }^{5}$ Department of Physics and Astronomy, Stony Brook University, Stony Brook, NY 11794-3800, USA \\ Received 2014 August 14; accepted 2015 June 15; published 2015 August 7
}

\begin{abstract}
The merger of binary neutron stars (BNSs) can lead to large amplifications of the magnetic field due to the development of turbulence and instabilities in the fluid, such as the Kelvin-Helmholtz shear instability, which drive small-scale dynamo activity. In order to properly resolve such instabilities and obtain the correct magnetic field amplification, one would need to employ resolutions that are currently unfeasible in global general relativistic magnetohydrodynamic simulations of BNS mergers. Here, we present a subgrid model that allows global simulations to take into account the small-scale amplification of the magnetic field which is caused by the development of turbulence during BNS mergers. Assuming dynamo saturation, we show that magnetar-level fields $\left(\sim 10^{16} \mathrm{G}\right)$ can be easily reached, and should therefore be expected from the merger of magnetized BNSs. The total magnetic energy can reach values up to $\sim 10^{51} \mathrm{erg}$ and the post-merger remnant can therefore emit strong electromagnetic signals and possibly produce short gamma-ray bursts.
\end{abstract}

Key words: gamma-ray burst: general - magnetohydrodynamics (MHD) - methods: numerical - stars: magnetars stars: neutron

\section{INTRODUCTION}

Binary neutron star (BNS) mergers are among the most powerful sources of gravitational waves (GWs) which are expected to be detected in the next few years by advanced LIGO and Virgo, and they are also the main candidates for the central engine of short gamma-ray bursts (SGRBs, e.g., see Berger 2014). The two main scenarios for the central engine of SGRBs involve the formation of a strongly magnetized torus around a spinning black hole (e.g., Rezzolla et al. 2011; Giacomazzo et al. 2013 and references therein) or the formation of a long-lived magnetar (e.g., Giacomazzo \& Perna 2013; Rowlinson et al. 2013). Neutron stars are often magnetized and, during the merger of two NSs, magnetic fields can be strongly amplified via instabilities in the plasma, such as the Kelvin-Helmholtz $(\mathrm{KH})$ instability (Price \& Rosswog 2006; Anderson et al. 2008; Baiotti et al. 2008; Giacomazzo et al. 2009, 2011). While previous Newtonian simulations have shown that magnetic fields can be amplified by several orders of magnitude, reaching magnetar-level fields of $\sim 10^{15} \mathrm{G}$ when starting with $\sim 10^{12} \mathrm{G}$ (Price \& Rosswog 2006), subsequent and independent studies in full general relativistic magnetohydrodynamics (GRMHD) did not find such large amplifications (Giacomazzo et al. 2009, 2011; Kiuchi et al. 2014). GRMHD simulations used grid-based codes with resolutions that were not sufficiently high to resolve the smallest turbulent scale and reach convergence. For example, in the presence of $\mathrm{KH}$ instabilities, higher resolutions can resolve smaller-scale vortices (Baiotti et al. 2008) and this can lead to stronger magnetic field amplifications. In addition, local high-resolution simulations performed in the last few years have shown that magnetic fields can indeed be amplified at merger by several orders of magnitude and reach equipartition with the kinetic energy of the turbulent fluid (Obergaulinger et al. 2010; Zrake \& MacFadyen 2013). Unfortunately, in order to fully resolve such scales, one would need to employ resolutions of the order of $\sim 0.1 \mathrm{~m}$ or higher (Obergaulinger et al. 2010; Zrake \& MacFadyen 2013), which is currently impossible to obtain in global simulations of BNS mergers (where resolutions are of the order of $\sim 100 \mathrm{~m}$ ).

Here, we develop a subgrid model which allows global GRMHD simulations of BNS merger to include small-scale effects and, in particular, properly resolve the magnetic field amplification during merger. Other subgrid models have already been used with success in other scenarios, such as magnetic field amplification in accretion disks (Sadowski et al. 2014), and here we show for the first time their useful role during BNS mergers.

Section 2 details our numerical methods and the initial models, and in Section 3 we provide a detailed description of the implementation of the subgrid model. Section 4 describes the evolution of the magnetic field when the subgrid model is implemented. In Section 5, we discuss whether this amplification is only localized in a small region of the domain or if it also happens on a global scale, while in Section 6 we discuss the magnetic energy generated by the turbulence and its impact on electromagnetic emission, including the possible production of SGRBs. Finally, Section 7 summarizes our main results. For convenience, we use a system of units in which $c=G=M_{\odot}=1$ unless explicitly stated otherwise.

\section{NUMERICAL METHODS AND INITIAL DATA}

The simulations presented here were performed using our fully GRMHD code Whisky (Giacomazzo \& Rezzolla 2007; Giacomazzo et al. 2011; Giacomazzo \& Perna 2013), which is coupled with the publicly available Einstein Toolkit (Löffler et al. 2012). In particular, the spacetime evolution is computed using the McLachlan code (Löffler et al. 2012) while Whisky solves the equations of GRMHD written in a conservative form (Giacomazzo \& Rezzolla 2007). In order to guarantee the divergence-free character of the magnetic field, 
we directly evolve the vector potential using the modified Lorenz gauge (Farris et al. 2012; Giacomazzo \& Perna 2013).

We also use adaptive mesh refinement via the Carpet driver (Löffler et al. 2012) by adopting six refinement levels with the finest grids completely covering each of the NSs. After the merger, the finest grid is enlarged in order to cover the hypermassive neutron star (HMNS; Giacomazzo et al. 2011). Our fiducial runs have a resolution of $\sim 225 \mathrm{~m}$ on the finest grid (see discussion in Section 4) while the coarsest grid extends up to $\sim 778 \mathrm{~km}$.

The initial data was produced using the publicly available code LORENE (Taniguchi \& Gourgoulhon 2002). The matter is modeled using a polytropic equation of state $(\mathrm{EOS}) p=K \rho^{\Gamma}$, where $p$ is the pressure, $\rho$ the rest-mass density, $K=123.6$, and $\Gamma=2$. An ideal-fluid EOS with $\Gamma=2$ is used during the evolution in order to allow for shock heating during the merger. The initial data is the same "high-mass" model used in our previous simulations (Baiotti et al. 2008; Giacomazzo et al. 2011; Rezzolla et al. 2011) and consists of an equalmass system of two NSs with gravitational mass ${ }^{6} \sim 1.5 M_{\odot}$ at an initial coordinate separation of $\sim 46 \mathrm{~km}$ (approximately 2.5 orbits before merger). The magnetic field is initially purely poloidal and aligned with the angular momentum of the binary as in Giacomazzo et al. (2011). The initial amplitude of the maximum of the magnetic field, as measured by a normal observer, is $\sim 2.5 \times 10^{12} \mathrm{G}$.

\section{THE SUBGRID MODEL}

Our subgrid model is intended to account for electromotive forces arising from unresolved fluctuations in the magnetic field and bulk fluid velocity. In particular, we assume that the unresolved turbulence, and the velocity field associated with it, gives rise to an extra electric field $\boldsymbol{E}_{\text {subgrid }}$ which is added to the right-hand side of the evolution equation for the vector potential $\boldsymbol{A}$ :

$$
\partial_{t} \boldsymbol{A}=-\boldsymbol{E}_{\text {ideal }}-\boldsymbol{E}_{\text {subgrid }},
$$

where $\boldsymbol{E}_{\text {ideal }}$ is the standard electric field coming from the idealMHD equations and is computed using the flux-CD approach as described in Giacomazzo et al. (2011). We note that this new evolution equation for the vector potential does not violate Maxwell's equations, but it may not satisfy the ideal-MHD condition. Our assumption is that $\boldsymbol{E}_{\text {subgrid }}$ would naturally arise in very high-resolution simulations where the turbulence is fully resolved, but that it is currently missing due to the still low resolution employed in BNS simulations. We therefore adopt a closure scheme intended to account for small-scale dynamo action driven by the turbulent cascade at the unresolved length scales. ${ }^{7}$ The effect of this dynamo has been found, through detailed local simulations (Zrake \& MacFadyen 2013) of relativistic MHD turbulence, to drive the largescale (resolved) magnetic energy density toward equipartition with the local turbulent kinetic energy density on a timescale given by the turnover of the energy containing eddies. We characterize the turbulent kinetic energy density by the field $\Delta w$, whose value is obtained through a prescription outlined

\footnotetext{
6 The gravitational mass is measured when the stars are isolated.

7 Such a closure scheme is not unique and a detailed numerical analysis with local simulations will be performed in a future paper in order to further assess the robustness of our subgrid model.
}

below. The exponentially growing solution is then given by

$$
\boldsymbol{E}_{\text {subgrid }}=-S_{\text {subgrid }} \boldsymbol{A}
$$

and parameterized around the exponentiation rate $S_{\text {subgrid }}$, which we take to be a fraction $c_{1}$ of the local fluid vorticity. Exponentiation of the magnetic field stops when the local electromagnetic energy density is a fraction $c_{2}$ of $\Delta w$. Thus, our closure scheme is parameterized around the following prescription for $S_{\text {subgrid }}$ :

$$
\begin{aligned}
S_{\text {subgrid }} \equiv & c_{1} \max \left(|\nabla \times v|-c_{3}, 0\right) \\
& \times \max \left(1-c_{4} \frac{\rho_{\text {atmo }}}{\rho}, 0\right) \\
& \times \max \left(1-\frac{b^{2}}{c_{2} \Delta w}, 0\right),
\end{aligned}
$$

where $\rho_{\text {atmo }}=10^{-10}$ is the value of the rest-mass density $\rho$ in the artificial atmosphere, $b^{2}$ is the magnetic energy density, and $\Delta w$ is a measure of the kinetic energy of the turbulent portion of the fluid (Duffell \& MacFadyen 2013). The coefficients $c_{1}=0.5, c_{2}=0.6, c_{3}=0.5$, and $c_{4}=10^{4}$ have been chosen based on the results of Zrake \& MacFadyen (2013; coefficients $c_{1}$ and $c_{2}$ ) and in order to avoid spurious magnetic field amplifications (coefficients $c_{3}$ and $c_{4}$ ), especially in regions near the surface of the neutron star where the (flat-space) vorticity, $|\nabla \times v|$, is artificially high because of a jump in the value of the velocity (since $v$ is set to zero in the artificial atmosphere). We note, in particular, that the value of $c_{2}$ sets the saturation level for the magnetic field and our value $\left(c_{2}=0.6\right)$ stops the magnetic field growth when the magnetic energy density is equal to $60 \%$ of the kinetic energy density, in agreement with the results of Zrake \& MacFadyen (2013). The coefficient $c_{1}$ was chosen instead to allow the growth to happen on a timescale of $\sim 1 \mathrm{~ms}$ (Zrake \& MacFadyen 2013). Note that the coefficients $c_{3}$ and $c_{4}$ instead need to be fine tuned in order to avoid spurious amplifications, especially at the NS surface. $\Delta w$ is computed following Duffell \& MacFadyen (2013) where for an ideal-fluid EOS we have $w \equiv \rho+p /(\Gamma-1)$, and hence (since $\Gamma=2$ in our case)

$$
\Delta w=\left(\langle\rho\rangle_{\mathrm{Cons}}+\langle p\rangle_{\mathrm{Cons}}\right)-\left(\langle\rho\rangle_{\mathrm{Vol}}+\langle p\rangle_{\mathrm{Vol}}\right),
$$

where $\langle\rho\rangle_{\text {Vol }}$ and $\langle p\rangle_{\text {Vol }}$ are the simple volume averages of the rest-mass density and pressure, respectively, while $\langle\rho\rangle_{\text {Cons }}$ and $\langle p\rangle_{\text {Cons }}$ are the "conservative" averages of the rest-mass density and pressure (see Duffell \& MacFadyen 2013 for details). The last two are computed by averaging the conserved variables over a region of space and then computing $\rho$ and $p$ using our standard conservative to primitive solver (Giacomazzo \& Rezzolla 2007). When computing the volume average and the conservative average of $\rho$ and $p$, one needs to choose the size of the volume over which the average is computed. In our simulations, we used a cubic box of size $6 \Delta x$, with $\Delta x$ being the resolution of the grid. In this way, when computing $\Delta w$ on each grid point, we simply need to know the values of the primitive and conservative variables on the next three grid points in each direction (e.g., the three grid points on the left and the three on the right along the $x, y$, and $z$ directions). This 


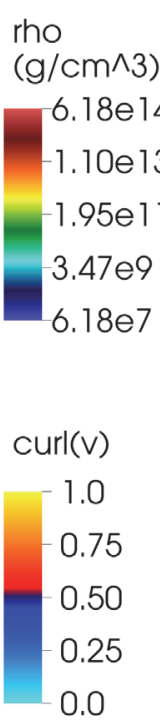

rho
$(\mathrm{g} / \mathrm{cm} \wedge 3)$
$6.18 \mathrm{e} 14$
$-1.10 \mathrm{e} 13$
$-1.95 \mathrm{e} 11$
$3.47 \mathrm{e} 9$
$6.18 \mathrm{e} 7$

S_subgrid
$(1 / \mathrm{ms})$
$2.03 \mathrm{e} 2$
-3.61
$6.4 \mathrm{e}-2$
$-1.14 \mathrm{e}-3$
$2.03 \mathrm{e}-5$

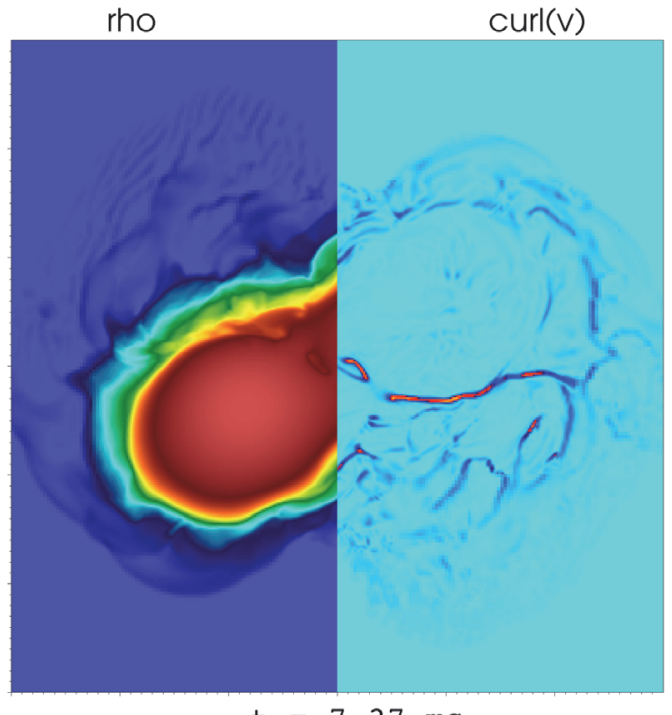

$t=7.37 \mathrm{~ms}$

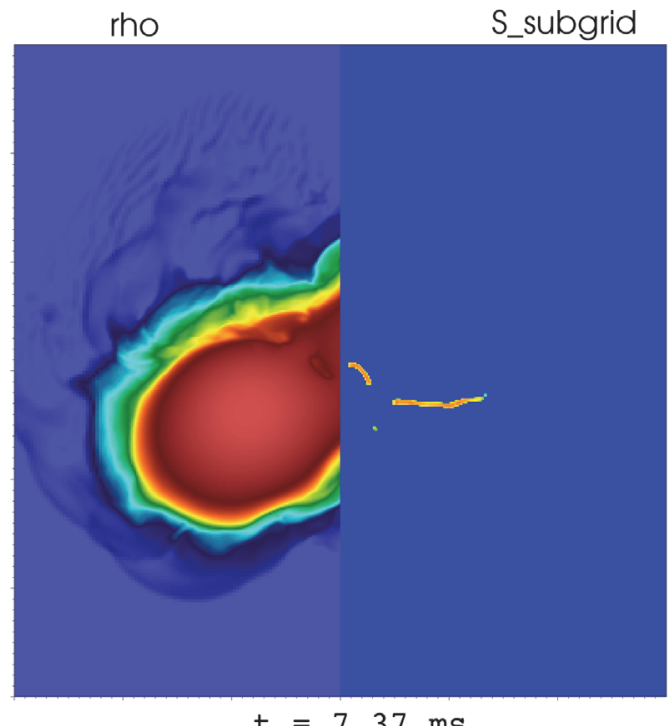

rho

$(\mathrm{g} / \mathrm{cm} \wedge 3)$

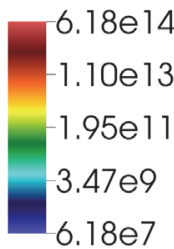

$6.18 \mathrm{e} 7$

delta_w

$(\mathrm{erg} / \mathrm{cm} \wedge 3)$

$5.55 e 33$

9.87 e31

$-1.76 e 30$

$3.12 \mathrm{e} 28$

$5.55 e 26$

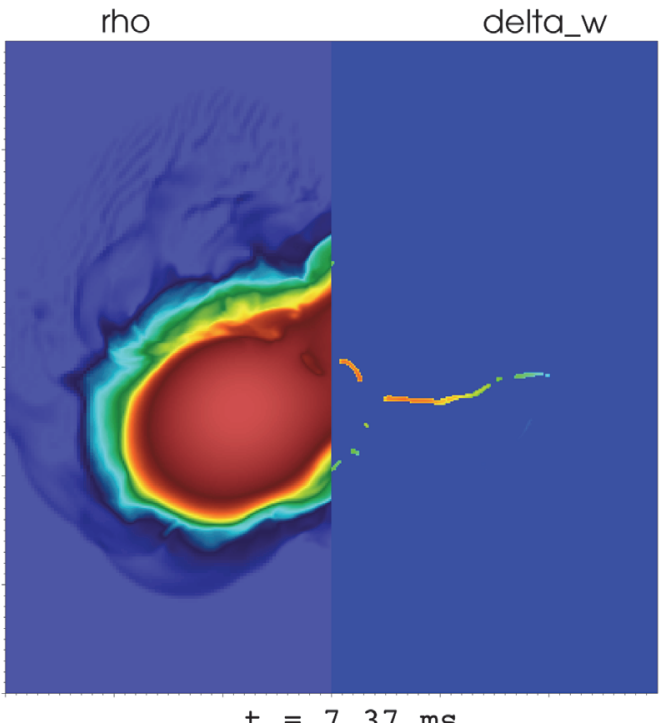

$\mathrm{t}=7.37 \mathrm{~ms}$

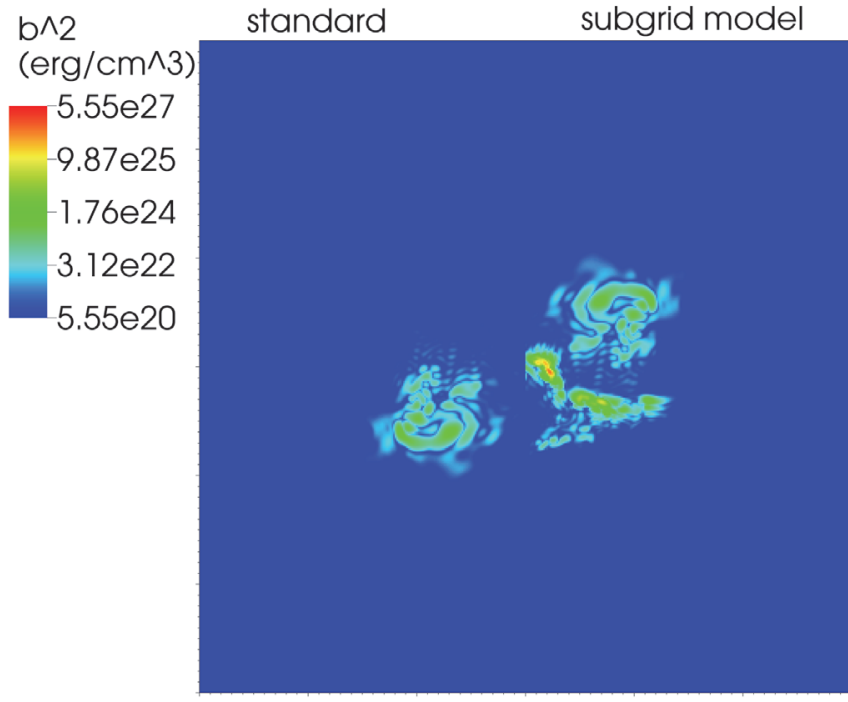

$t=7.37 \mathrm{~ms}$

Figure 1. First three panels show on the left side the value on the $x y$ equatorial plane of the rest-mass density $\rho$ (in $\mathrm{g} \mathrm{cm}^{-3}$ ) and on the right side the value of $|\nabla \times v|$ in geometric units (top left panel), of $\Delta w$ in erg $\mathrm{cm}^{-3}$ (top right panel), and of $S_{\text {subgrid }}$ in $\mathrm{ms}^{-1}$ (bottom left panel). They all refer to our fiducial run with the subgrid model. The subgrid model in our runs is applied in those regions in which the vorticity $|\nabla \times v|$ is larger than $c_{3}=0.5$ (in geometric units) and $\Delta w$ for computational reasons is calculated only in those regions. The bottom right panel shows instead the magnetic energy density $b^{2}$ in erg $\mathrm{cm}^{-3}$ for a "standard" evolution (left side) and for the case in which the subgrid model is implemented (right side). All panels refer to time $t \sim 7.4 \mathrm{~ms}$ and they cover the region between $\sim-45 \mathrm{~km}$ and $\sim 45 \mathrm{~km}$ in both $x$ and $y$. These four panels show that our subgrid model amplifies the magnetic field mainly in the region near the center (where the KH instability is more active; e.g., see Baiotti et al. 2008) and excludes low-density zones near the artificial atmosphere.

choice has been made in order to reduce the computational cost (i.e., MPI calls) of such computations.

\section{MAGNETIC FIELD AMPLIFICATION AND SATURATION}

We ran two simulations using the same initial data, but in one case we used the subgrid model described in the previous section, while in the other we evolved the system using the "standard" vector potential equations, i.e., without adding the subgrid term $S_{\text {subgrid }} A_{i}$ to the right-hand side of the evolution equations for $A_{i}$. Here, we first report on the results obtained with the new subgrid model and in the next section we compare with the "standard" evolution.
In Figure 1, we show, at $t \sim 7.4 \mathrm{~ms}$, the value on the equatorial plane of the rest-mass density $\rho$, of $|\nabla \times v|$ (top-left panel), of $\Delta w$ (top-right panel), of $S_{\text {subgrid }}$ (bottom-left panel), and of the magnetic energy density $b^{2}$ (bottom-right panel). In the last panel, in particular, we compare the magnetic energy density between a "standard" evolution (left side) and the case in which the subgrid model is implemented (right side). From these figures one can see that the regions where $S_{\text {subgrid }}$ is non-zero and the magnetic field is amplified are indeed those where the $\mathrm{KH}$ instability is more active (compare also with Price \& Rosswog 2006 and Baiotti et al. 2008). Indeed, in those regions, both the vorticity $(|\nabla \times v|)$ and $\Delta w$ are much larger than zero and have their maximum values. Note also that the vorticity is also quite large in regions outside the central 


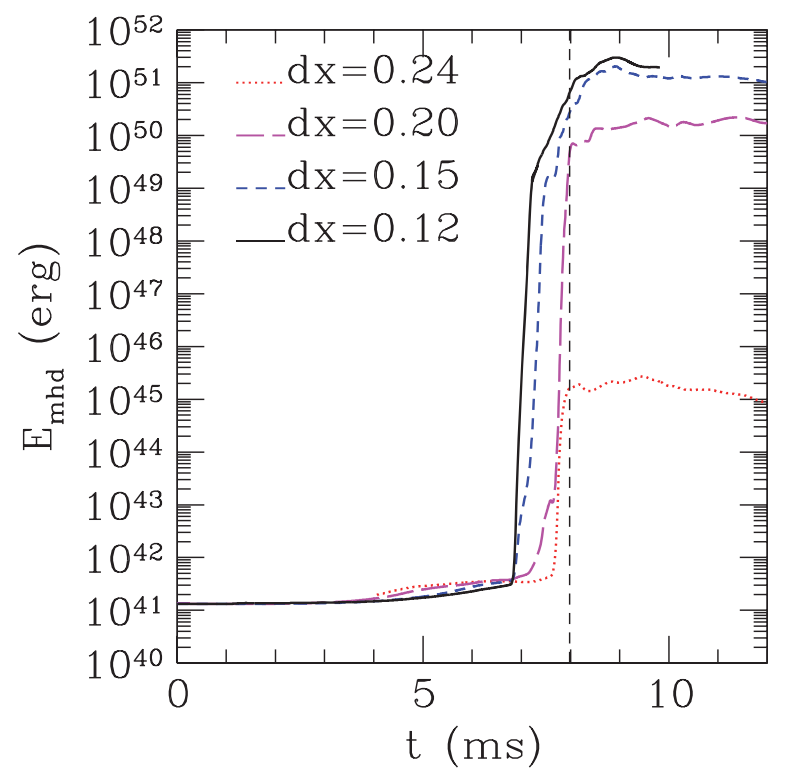

Figure 2. Evolution of the total magnetic energy when the subgrid model is implemented and for different resolutions: the solid black line refers to our highest-resolution run with $\Delta x=0.12 \approx 180 \mathrm{~m}$, the blue dashed line to $\Delta x=0.15 \approx 220 \mathrm{~m}$, the purple long dashed line to $\Delta x=0.20 \approx 300 \mathrm{~m}$, and the red dotted line to $\Delta x=0.24 \approx 360 \mathrm{~m}$ (all resolutions $\Delta x$ refer to the resolution on the finest grid, i.e., the one covering the NSs). The vertical black dashed line indicates the time of the merger of the two NS cores (which also corresponds to the maximum amplitude of the gravitational wave signal), while the external layers start merging a few ms before.

region. The choice of parameters in Equation (3) is such that those regions are excluded, since the turbulence there, which is anyway smaller than in the central regions, is due to the interaction with the artificial atmosphere.

In Figure 2, we show how the amplification changes with resolution. We reran the same model with one higher resolution $(\Delta x=0.12 \approx 180 \mathrm{~m})$ and two lower resolutions $(\Delta x=0.20 \approx 300 \mathrm{~m}$ and $\Delta x=0.24 \approx 360 \mathrm{~m})$. In Figure 2 , we plot the evolution of the magnetic energy and, while the lowest-resolution run (red dotted line) shows only a modest increase due to just two orders of magnitude amplification in the magnetic field, the other three resolutions show a much larger increase. In particular, the two highest-resolution runs produce the same magnetic energy (and the same magnetic field values), indicating that saturation has been reached. We note that this is the first time that such a saturation level is reached in a BNS simulation. Previous GRMHD simulations were not able to amplify the magnetic field more than $\sim 1$ order of magnitude at merger and only the Newtonian simulations by Price \& Rosswog (2006) showed large magnetic field amplifications, but no saturation was reached and different values were obtained for different resolutions.

\section{LOCAL OR GLOBAL MAGNETIC FIELD AMPLIFICATION?}

In Figure 3, we plot the weighted average of the magnetic field amplitude:

$$
B_{\text {mean }} \equiv \frac{\int \rho B d V}{\int \rho d V},
$$

with $d V$ being the proper volume. The black solid lines represent the evolution of $B_{\text {mean }}$ when the subgrid model is

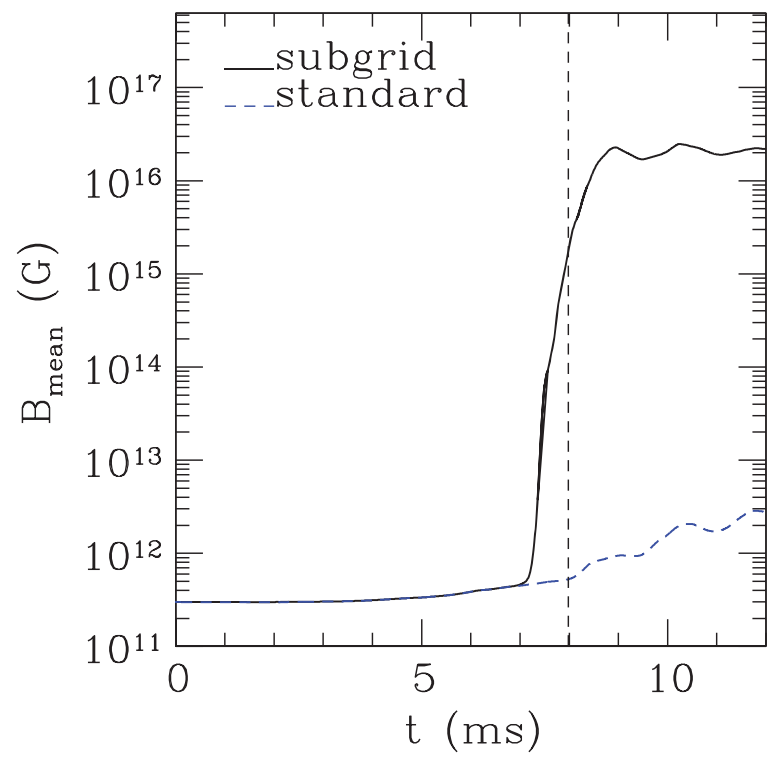

Figure 3. Evolution of the mean value of the magnetic field when the subgrid model is implemented (black solid line) and when it is not (blue dashed line). The vertical dashed line shows the time of merger (when the NS cores collide). While in a "standard" simulation, i.e., a simulation where the subgrid model is not implemented, the magnetic field grows by only $\sim 1$ order of magnitude, in the simulation implementing the subgrid model the magnetic field grows up to $\sim 10^{16} \mathrm{G}$ and it saturates when reaching equipartition with the kinetic energy of the fluid in the turbulent regions.

used, while the blue dashed line represents the "standard" evolution. In both cases, we used our fiducial resolution $(\Delta x=0.15 \approx 220 \mathrm{~m})$. First of all, while the maximum of the magnetic field saturates to $\sim 10^{17} \mathrm{G}$ when the subgrid model is used, its mean value saturates to $\sim 10^{16} \mathrm{G}$. This is a clear indication that during the evolution the strong magnetic field generated in the turbulent regions expands and covers a large portion of the HMNS formed after the merger. The magnetic field amplification is therefore not killed during the merger but survives and may considerably affect the post-merger evolution (Giacomazzo et al. 2011). The blue dashed line represents instead the mean value of the magnetic field when the subgrid model is not used. In this case, the magnetic field grows only by one order of magnitude, as seen in previous simulations (Giacomazzo et al. 2009, 2011; Rezzolla et al. 2011; Kiuchi et al. 2014). By properly taking into account the amplifications due to the subgrid-scale turbulence, the magnetic field is amplified by $\sim 4$ orders of magnitude with respect to what can be afforded by current resolutions. Indeed, we expect that even without our subgrid model, one should be able to obtain such large fields when employing sufficiently large resolutions in order to reach saturation (which may not happen for $\Delta x \gtrsim 0.1 \mathrm{~m})$.

\section{MAGNETIC ENERGY AND GAMMA-RAY BURST ENGINE}

In Figure 4, we plot the evolution of the magnetic energy both when the subgrid model is used (solid black line) and when it is not (dashed blue line). We also plot the turbulent energy $E_{\Delta w}$, i.e., the integral of $\Delta w$ for the case in which the subgrid model is used. During the merger, $E_{\Delta w}$ grows by several orders of magnitude and is followed by an increase in 


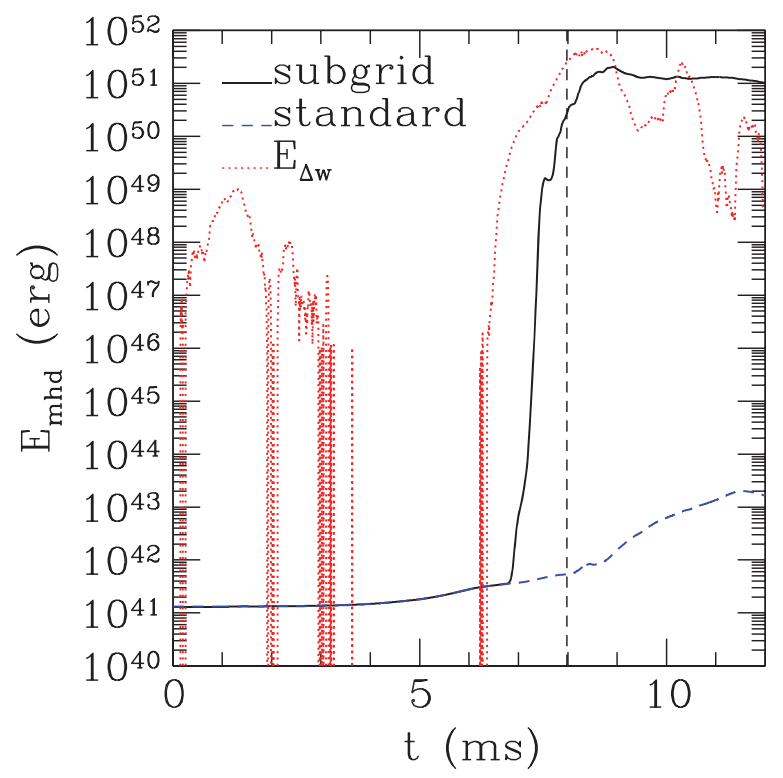

Figure 4. Evolution of the magnetic energy when the subgrid model is implemented (black solid line) and when it is not (blue dashed line). The vertical black dashed line represents the time of merger of the two NS cores. The red dotted line represents instead the integral of $\Delta w$ computed where $S_{\text {subgrid }}>0$. The values of $E_{\Delta w}$ at $t<4 \mathrm{~ms}$ are due to the artificial shocks that develop on the NS surfaces during the inspiral (due to the fact that we evolve our NSs using an ideal-fluid EOS and that our NSs do not have a solid crust). As one can easily see, the values of $E_{\Delta w}$ during the first part of the inspiral are at least $\sim 2$ orders of magnitude below those reached during merger. Moreover, they do not affect the evolution of the magnetic field as one can see both from this figure (the magnetic energy is constant as in the standard case) and from Figure 3, where the mean value, as well as the maximum (not shown), of the magnetic field does not grow during the inspiral and it is identical to the value in the standard run (i.e., when the subgrid model is not used).

the magnetic energy. The small delay (less than a ms) between the onset of the two growths is due to the time the magnetic field needs in order to be amplified over a large region (the maximum of the magnetic field grows indeed much earlier and almost simultaneously with the growth of $E_{\Delta w}$ ). These results predict the rapid production of a magnetic energy reservoir on the order of $\sim 10^{51} \mathrm{erg}$ in the moments after the merger onset. It was pointed out in Zrake \& MacFadyen (2013) that a powerful electromagnetic transient could be powered by the conversion of even a small fraction of that magnetic energy into escaped photons. There, it was proposed that strong magnetic fields undergoing violent reconnection in the merger remnant's atmosphere could power electromagnetic explosions capable of accelerating plasma to large Lorentz factors. High-energy photons escape to the observer once the accelerated plasma has overtaken the slow $(\sim 0.1 c)$ baryonic outflow. The production of magnetic explosions is proposed to operate in a manner not unlike those thought to power the so-called giant magnetar flares (e.g., Thompson \& Duncan 1995). These high-energy transients could be associated with SGRB precursor emission (Troja et al. 2010) or even the SGRB itself.

\section{SUMMARY}

We presented the first subgrid model for GRMHD simulations of BNS mergers that can be used to study small-scale magnetic field amplifications in global BNS simulations. We show for the first time that, by assuming dynamo saturation, magnetic field values of the order of $\sim 10^{16} \mathrm{G}$ can be easily produced in BNS mergers. This large magnetic field corresponds to a magnetic energy in the plasma of $\sim 10^{51} \mathrm{erg}$. Such large magnetic fields and energy can lead to the production of strong electromagnetic signals (Siegel et al. 2014), SGRBs (Rezzolla et al. 2011; Giacomazzo \& Perna 2013), and also long-lasting GW emission if a stable magnetar is formed after merger (Dall'Osso et al. 2015). In future papers, we will study the effect of this field amplification on the evolution of the HMNS, on the shape of the GW signal, on the possible emission of relativistic jets, and on ejecta masses and distribution, which may have consequences for $r$-process nucleosynthesis as well as jet collimation and the production of electromagnetic transients from circum-merger interactions (Nakar \& Piran 2011; Metzger \& Berger 2012).

As a final comment, we also note that this subgrid model should be implemented only as long as GRMHD simulations of BNSs are not able to use the resolutions required to fully resolve the turbulence. This will require high-order numerical codes combined with very high resolutions $(\sim 0.1 \mathrm{~m})$ which are currently unfeasible.

We acknowledge Zachariah Etienne and an anonymous referee for useful comments and suggestions. B. G. and R. P. acknowledge support from NSF grant No. AST 1009396 and NASA grant No. NNX12AO67G. B. G. also acknowledges support from MIUR FIR grant No. RBFR13QJYF. This research was also supported in part by NASA through Fermi grant NNX13AO93G and by the NSF through grant AST1009863. This work used XSEDE (allocation TGPHY110027) which is supported by NSF grant No. OCI1053575.

\section{REFERENCES}

Anderson, M., Hirschmann, E. W., Lehner, L., et al. 2008, PhRvL, 100, 191101

Baiotti, L., Giacomazzo, B., \& Rezzolla, L. 2008, PhRvD, 78, 084033

Berger, E. 2014, ARA\&A, 52, 43

Dall'Osso, S., Giacomazzo, B., Perna, R., \& Stella, L. 2015, ApJ, 798, 25

Duffell, P. C., \& MacFadyen, A. I. 2013, ApJ, 775, 87

Farris, B. D., Gold, R., Paschalidis, V., Etienne, Z. B., \& Shapiro, S. L. 2012, PhRvL, 109, 221102

Giacomazzo, B., \& Perna, R. 2013, ApJL, 771, L26

Giacomazzo, B., Perna, R., Rezzolla, L., Troja, E., \& Lazzati, D. 2013, ApJL, 762, L18

Giacomazzo, B., \& Rezzolla, L. 2007, CQGra, 24, S235

Giacomazzo, B., Rezzolla, L., \& Baiotti, L. 2009, MNRAS, 399, L164

Giacomazzo, B., Rezzolla, L., \& Baiotti, L. 2011, PhRvD, 83, 044014

Kiuchi, K., Kyutoku, K., Sekiguchi, Y., Shibata, M., \& Wada, T. 2014, PhRvD, 90, 041502

Löffler, F., Faber, J., Bentivegna, E., et al. 2012, CQGra, 29, 115001

Metzger, B. D., \& Berger, E. 2012, ApJ, 746, 48

Nakar, E., \& Piran, T. 2011, Natur, 478, 82

Obergaulinger, M., Aloy, M. A., \& Müller, E. 2010, A\&A, 515, A30

Price, D. J., \& Rosswog, S. 2006, Sci, 312, 719

Rezzolla, L., Giacomazzo, B., Baiotti, L., et al. 2011, ApJL, 732, L6

Rowlinson, A., O’Brien, P. T., Metzger, B. D., Tanvir, N. R., \& Levan, A. J. 2013, MNRAS, 430, 1061

Sadowski, A., Narayan, R., Tchekhovskoy, A., et al. 2014, MNRAS, 447, 49

Siegel, D. M., Ciolfi, R., \& Rezzolla, L. 2014, ApJL, 785, L6

Taniguchi, K., \& Gourgoulhon, E. 2002, PhRvD, 66, 104019

Thompson, C., \& Duncan, R. C. 1995, MNRAS, 275, 255

Troja, E., Rosswog, S., \& Gehrels, N. 2010, ApJ, 723, 1711

Zrake, J., \& MacFadyen, A. I. 2013, ApJL, 769, L29 